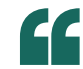

The major drawback [with $\mathrm{Sml}_{2}$ ] has always been the need to use superstoichiometric amounts of the reagent, raising issues of both cost and waste gु

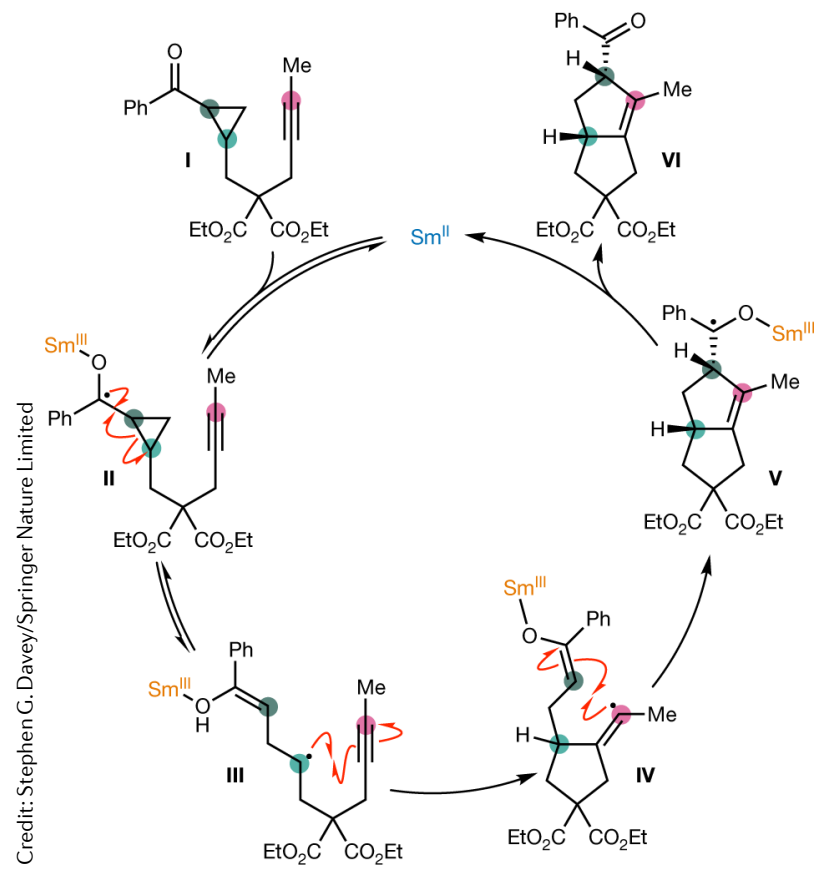

Samarium diiodide has been used as a single-electron transfer (SET) reagent for over 40 years. Now, writing in Nature Catalysis, David Procter and co-workers from the University of Manchester have shown how it can be used in substoichiometric amounts to catalyse radical cyclization cascades to produce complex bicyclic and tricyclic structures.

A unique combination of high reactivity and high selectivity means that radical cyclization reactions have always been a popular way to access complex polycyclic structures. Such structures are found in many biologically active molecules such as

RADICAL REACTIONS

\title{
Catalytic Kagan
}

natural products and drugs. Of particular recent interest has been their application in the development of cascade reactions that enable the rapid generation of molecular complexity from simple starting materials. "Thousands of papers have reported on the synthetic applications of samarium diiodide (often known in this context as Kagan's reagent); it's the go-to reagent for reductive, radical chemistry," says Procter. "The major drawback has always been the need to use super-stoichiometric amounts of the reagent, raising issues of both cost and waste." Waste can be reduced by developing catalytic versions of known stoichiometric reactions and as a result is one of twelve principles of green chemistry.

Procter and co-workers are not the first to attempt to address the problem of catalysis with $\mathrm{SmI}_{2}$. However, previous approaches have usually relied on the addition of a large excess of inexpensive co-reductants to regenerate $\mathrm{Sm}^{\text {II }}$ from Sm ${ }^{\text {III }}$.At the outset of this work," explains Procter, "we asked ourselves one question: can reactions catalytic in $\mathrm{SmI}_{2}$, without a co-reductant, be developed by careful design of the reaction mechanism?"

Procter and co-workers reaction design begins with a cyclopropyl substituted ketone I that they describe as spring-loaded. SET from the $\mathrm{Sm}^{\text {II }}$ catalyst gives a ketyl radical II. Cyclopropyl ring-opening leads to a new radical III that undergoes ringclosing with a pendant radical trap (in this case an alkyne) to give IV. A second ring-closure generates a ketyl radical $\mathbf{V}$ that can then return an electron to samarium, affording the product VI and returning the catalyst to enable turnover of the catalytic cycle. The reaction scope includes reactions with alkenes, alkynes and even some de-aromatizing cyclizations with benzofurans and benzothiophenes.

Mechanistic studies support the catalytic cycle proposed. Among other experiments, the possibility of a Lewis acid-catalysed process was eliminated when no products were obtained using a variety of other Lewis acids and reaction using samarium was suppressed by the addition of a radical inhibitor. Alternative SET reagents also gave low yields, which favours turnover by electron transfer to regenerate $\mathrm{Sm}^{\mathrm{II}}$ rather than a chain process in which an electron is transferred directly from the final ketyl radical intermediate $\mathbf{V}$ to a second molecule of starting material I.

"We believe that by adapting our radical relay strategy, other widely used radical processes, closely associated with the ubiquitous reducing agent, can be rendered catalytic in $\mathrm{SmI}_{2}$," says Procter.

Stephen G. Davey

ORIGINAL ARTICLE Huang, H.-M. et al. $\mathrm{Sml}_{2}$-catalysed cyclization cascades by radical relay. Nat. Catal. https://doi.org/10.1038/s41929018-0219-x (2019)

FURTHER READING Plesniak, M. et al. Radical cascade reactions triggered by single electron transfer. Nat. Rev. Chem. 1, 0077 (2017) 